\title{
Intangibile, Emergente, Esplicito. II progetto di paesaggio per la transizione ecologica
}

\author{
Ludovica Marinaro \\ Università di Modena e Reggio Emilia (UNIMORE), Italia \\ ludovica.marinaro@unimore.it
}

\begin{abstract}
Quello che si aprirà a seguito del processo di transizione ecologica oggi altro non è che un paesaggio intangibile. Ancora utopico, viene pensato come depositario dei nostri desiderata, espressione comunitaria di una tecnologia democratica, dimensione flessibile che finalmente potrà accogliere una società ravveduta. In quanto ancora potenziale e strettamente dipendente dal nostro agire presente, il paesaggio intangibile della transizione ecologica, possiede altresì il suo yang: il paesaggio del riscaldamento globale di $2^{\circ} \mathrm{C}$, nello scenario ipotizzato dall'ultimo report dell'Intergovernamental Panel on Climate Change (IPCC). In bilico, vi è oggi bisogno di strategie efficaci per rendere concreto l'obiettivo della transizione ed il paesaggio più che mera destinazione può oggi essere inteso come attore principale sulla scena urbana. Sgombrato il campo da equivoci e mistificazioni, il saggio esplora le potenzialità del progetto di paesaggio come progetto politico di una transizione solidale oltre che ecologica.
\end{abstract}

\section{Parole chiave}

Paesaggi intangibili, transizione ecologica, progetto di paesaggio, paesaggi emergenti, politica

\begin{abstract}
The result of the ecological transition process today is nothing more than an intangible landscape. Still utopian, it is thought of as the custodian of our desires, the communitarian expression of a democratic technology, a flexible dimension that will finally welcome a reformed society. As still potential and strictly dependent on our present action, the intangible landscape of ecological transition also has its Yang: the landscape of global warming of $2^{\circ} \mathrm{C}$, in the scenario hypothesized by the latest report of the Intergovernmental Panel on Climate Change (IPCC). In balance between catharsis and catastrophe there is now a need for effective strategies to make the objective of transition concrete and the landscape, more than a mere destination, can today be understood as the main actor on the urban scene. Having cleared the field of misunderstandings and mystifications, the essay explores the potential of the landscape project as a political project of a solidary as well as ecological transition.
\end{abstract}

\section{Keywords}

Intangible landscapes, ecological transition, landscape project, emergent landscapes, politics 


\section{Nóotos}

"Emerging landscapes are becoming brand new actors on political stage"

A Landscape Manifesto

Diana Balmori

In questi odierni hard times la netta sensazione di trovarsi dinanzi a un bivio cruciale tra catarsi e catastrofe potrebbe a ben vedere assalire chiunque e mentre in realtà ci troviamo parecchi passi più in là della biforcazione, nel bel mezzo di una "selva selvaggia e aspra e forte" "1, l'Europa è corsa ad armarsi delle necessarie mappe e della bussola e, dalla ratifica del primo accordo universale ${ }^{2}$ giuridicamente vincolante sul clima a livello mondiale nel 2016, con il Green Deal Europeo ${ }^{3}$ ha stabilito una rotta: quella della transizione ecologica, che vuol essere al contempo "giusta e inclusiva" (EC, 2019).

In viaggio, tutta la società nelle sue liquide sfaccettature prova ad aprirsi dei varchi. Distinti dalle file dei catastrofisti e dalle loro litanie, si riconoscono i visi compassati dei decrescisti felici ${ }^{4}$, quelli concentrati dei ligi, quelli ignari degli ultimi, scoppietta la goliardia degli scettici mentre fioriscono le espressioni esasperate di quella parte di élite culturale che ancora si interroga su chi effettivamente li rappresenti, fino ai negazionisti che inerti, arroganti e chiassosi chiudono la carovana. Vi sono poi i paesaggisti che appartengono ad una categoria ibrida di visionari con i piedi per terra, tra i pochi a 'saper vedere' la selva nella sua dimensione sensibile oltre che allegorica, sensibili alla $\Phi v ́ \sigma ı \varsigma^{5}$ e alla sua manifestazione tangibile. Di quel selvatico, pur nella sua acidità, nella bruttezza ${ }^{6}$ comunemente percepita e nel- la sua scomposta prorompenza, sanno apprezzare il peculiare dinamismo, assecondarlo per trarne vantaggio, interpretarne i segni e trarne insegnamento, cosicché l'individuazione del cammino per loro scaturisce semplicemente dall'osservazione e dal dialogo con il luogo, non dalla contrapposizione a esso ma sua naturale conseguenza, così il moto dalla stasi, l'emozione dall'estasi, il camminare dallo stare. Questo dialogo e progressiva domesticazione, cambia volto alla selva, aggiunge valore, le conferisce nuova dignità. Così la selva si dischiude, offre dei frutti, mostra un sentiero. Alcuni intorno se ne avvedono. La rivoluzione sembra tanto piccina quanto è prorompente. Ecco che il paesaggio della transizione ecologica si avvicina, rivelando una forma relazionale complessa che sovverte la logica lineare con la quale era pensato fino a quel momento. Quel paesaggio (della transizione ecologica) perde le sembianze di locus amoenus distante, mero culmine concreto e perfetto di un processo teleologico, e riscatta la sua natura processuale, testimonia il suo 'essere già qui' in forma intangibile e quindi la sua capacità di permeare del suo potenziale e dei suoi valori anche quei territori che sembravano essere esclusi da questo processo di conversione, perché ritenuti banali o brutti. Ciò equivale a riconoscere alla selva lo status di paesaggio e con essa a tutto il territorio, applicando ovvero uno dei principi rivo- 

selvaggia e aspra e forte" volti di territori complessi, talvolta sfuocati, talvolta eccessivamente saturi. Il paesaggio della transizione ecologica più che mera destinazione può e deve oggi essere inteso come attore principale, presente sulla scena urbana. In quanto paesaggio intangibile, si dà in forma di tensione ed è capace di crear movimento, di emozionare nel senso più complesso e profondo del termine ovvero quello di produrre un'attrazione a sé oltre che di coinvolgere e appassionare gli attori alla sua realizzazione.

Condiviso l'obiettivo prioritario della transizione ecologica dell'economia mondiale e nella convinzione che esso fosse già in nuce nella CEP il saggio inizia ad esplorare la potenzialità del progetto di paesaggio per realizzare la transizione a partire da una riflessione sul modo di intendere il paesaggio stesso e di includerlo all'interno della politica, come espresso dalla CEP, non già e non solo attraverso norme e leggi, ma ancor prima come 'principio', come approccio all'osservazione, interpretazione e innovazione della realtà la realtà che ci circonda per ripensare la città del XXI Secolo.

Intangibile, emergente, esplicito sono dunque le tre condizioni di cui intendo parlare, tre momenti di un ciclo programmatico-evolutivo del paesaggio che abitare sostenibile, di una condizione di nuovo equilibrio sistemico sul pianeta.

\section{Intangibile.}

\section{Cos'è la selva? Riconoscerla, orientarsi}

II presente ci chiama a coniugare le trasformazioni del territorio sovvertendo la logica che le ha sottese nella prassi comune fino ad oggi. Le operazioni che riguardano il paesaggio devono essere spogliate dal gravoso manto della 'mitigazione', vessillo di una prassi progettuale che sembra non riuscire a smarcarsi da un atavico senso di colpa ${ }^{10}$ legato alla trasformazione e all'introduzione del nuovo nei paesaggi consolidati della nostra penisola, all'adattamento alle mutate esigenze del nostro tempo. Come ulteriore conseguenza, perdura la vana convinzione che tutelare significhi immobilizzare il tangibile, senza avvedersi che ciò tende ad ingessare anche l'immaginario perché effetto di una visione incapace di accogliere la realtà come incessante divenire, come insieme di relazioni dinamiche il cui equilibrio è frutto di una danza "questa discesa nelle forme empatiche di relazione dentro e fuori di noi, tra gesto e cose, natura e oggetti, dinamica e spazio, scavo e sottosuolo, -che (ndr)restituisce un corpo quale soglia tra un equilibrio e l'altro" (Sieni, 2021). Tutelare significa prendersi cura di quei corpi e sottintende una relazione attiva, eloquente che parte dalla conoscenza e inevitabilmente tramuta i soggetti coinvolti.

Quando si parla di paesaggio però, il recinto che separa l'accademia dall'agone della pianificazione ordinaria e dalla prassi progettuale sembra ergersi ancora ben saldo. Generalizzando, abbiamo un problema di comunicazione con la politica e il governo del territorio: ciò è lampante dalla mancata attuazione della Convenzione Europea del Paesaggio, ratificata in Italia ormai quindici anni fa (2006) e disattesa, dal ritardo nella predisposizione dei piani paesaggistici normati dal Codice $^{11}$, che già peraltro soffre di una certa obsolescenza e necessiterebbe di essere attualizzato nel suo stesso impalcato di principi, 
dall'assenza del paesaggio all'interno dello PNIEC ${ }^{12}$ e, non ultimo, dalla recente diatriba circa il processo di transizione energetica, rispetto al quale si è addirittura andata profilando la situazione paradossale per cui la tutela del paesaggio possa confliggere con l'esigenza stringente della transizione all'utilizzo delle fonti di energia rinnovabili (FER).

"Per le soprintendenze l'eolico e il solare sui tetti sono brutti, degradano il paesaggio e quindi vengono bocciati. Gli esempi sono innumerevoli, da nord a sud, da edifici agricoli a brutte palazzine costruite nel dopoguerra il cui tetto è visibile da un edificio vincolato. La tesi che viene sostenuta ha anche un suo fondamento teorico, il compito loro assegnato per legge è quello di tutelare un valore primario che ha un riferimento stampato nell' articolo 9 della Costituzione Italiana. Spetta ad altri trovare risposte all'emergenza climatica, salvo non vadano in conflitto con il paesaggio. (...) Da una situazione di questo tipo si potrà uscire solo alzando il livello del confronto, coinvolgendo anche quel Ministero nel trovare soluzioni alla sfida di integrare i progetti nei paesaggi con criteri di valutazione e procedure trasparenti, che coinvolga icittadini" (Zanchini, 2021).

Così tuonava il vicepresidente di Legambiente, Edoardo Zanchini, sulle pagine del quotidiano Domani il 2 marzo 2021, a circa 15 giorni dall'istituzione del Ministero della Transizione Ecologica da parte del neo governo Draghi. Zanchini ha ragione: Ia transizione ecologica passa dal Ministero della Cultura. II fatto sconcertante è però che la "tutela del paesaggio" di cui parla l'articolo 9 della nostra Costituzione non ha nulla a che vedere con l'impostazione rigida e riduzionista del MiBACT, poiché implica ontologicamente l'innovazione (e l'ecologia aggiungo). E allora non è accettabile oggi sentir dire che le risposte all'emergenza climatica possano essere aprioristicamente in conflitto con il paesaggio, quando il paesaggio dovrebbe essere il luogo di mediazione dei conflitti, quando il paesaggio è altro, è di più rispetto a quanto il Codice sancisce. Si tratta di una condizione così intrisa di contraddizioni e non-sense alla quale è più che mai necessario porre rimedio ripar- tendo dai principi e da come essi si traducono nel linguaggio comune e nel comune agire territoriale ${ }^{13}$. Questo perché se il processo di transizione ecologica perdesse il suo dato paesaggistico, se lasciasse indietro la complessa dimensione intangibile del paesaggio relegandolo a questione meramente epidermica, visuale, allora avremo veramente perso una grande occasione, si rischierebbe per le giovani generazioni un processo ancor più incisivo e spietato di quel "depaesaggiamento" di cui parlava Alain Roger (1997), con tutte le sue conseguenze culturali ma anche economiche!

Ancora troppo diffusa è invece la convinzione che il progetto di paesaggio equivalga ad un non ben identificato 'progetto del verde', una sorta di panacea da somministrare ex-post per camuffare e nascondere le nuove trasformazioni, siano esse infrastrutture o architetture. Nell'opera di Ugo La Pietra (2015) questa convinzione comune che "il verde risolva" viene osservata e scandagliata attraverso una serie poetica di fotomontaggi, disegni e collage che mostrano l'evoluzione del verde nello spazio urbano dagli anni '80 sino ai giorni nostri. Questo libricino, che raccoglie il frutto di una ricerca provocatoria dell'artista durata decenni, in maniera molto ironica ed elegante ci mette di fronte alle controversie della percezione del rapporto essere umano-natura nella città, evidenziandone il ridicolo. Attraverso un inventario creativo degli spazi e dei modi in cui il 'verde' è stato relegato in ambito urbano, il designer milanese, ci pone da un lato di fronte all'assurdità, alla presunzione, alla negligenza e all'ingenuità con la quale abbiamo nel tempo trattato la natura nelle nostre città, dall'altro fornisce attraverso il disegno (e il progetto) delle suggestioni per riportare la spontaneità della natura al centro di una visione progettuale urbana. La sineddoche Verde=Natura è di fatto un'ulteriore prova che ci fornisce il linguaggio di quello svilimento, un tentativo di appiattire la complessità della natura per poterla meglio controllare, addomesticare, piegarla al servizio dei bisogni antropici. 
Il fatto che poi questa semplificazione sia avvenuta a favore del piano sensoriale della vista e per di più del dato cromatico è indicativo di una cultura, la nostra, dominata dal senso della vista (Venturi Ferriolo, 2009; Pallasmaa, 2005) e dalla preponderanza dell'immagine a tal punto da aver pervaso anche i campi dell'urbanistica e dell'architettura. Nella prima legge italiana (DM 1444 del 2 Aprile 1968) che introduce gli standard urbanistici per la città moderna si parla di "verde pubblico" e non già di "natura pubblica' ed anche la strategia Europea per la Transizione Ecologica si chiama "Green Deal". Anche la disciplina del paesaggio nel nostro Paese non è stata e non è tuttora esente da questa tacita influenza. Sappiamo bene quanto spesso ancora si confonda o si voglia ridurre la questione della compatibilità e dell'integrazione del nuovo nei paesaggi con mere questioni di adattamento cromatico, con una spiccata comune propensione per l'uso del verde, appunto, cosicché di fronte al problema progettuale dell'inserimento paesaggistico di una nuova opera, sia una piccola pergola o una nuova porzione di infrastruttura energetica, tra le proposte normalmente più gettonate si sente: "Dipingiamolo di verde!". Tuttavia Inserire non significa Mimetizzare, ed Integrare non significa Nascondere. Vi è oggi più che mai la necessità di disambiguare e restituire alle parole la propria forza e profondità semantica. II Paesaggio ha poco a che fare con il camouflage. Inteso secondo i principi che emanano dalla CEP e che ancor prima affondano le radici nel pensiero ecologico da Bateson a McHarg, il progetto di paesaggio è un'azione rivelatrice e non un meccanismo di creazione dell'occulto, non deve ovvero contribuire a creare "cose che siano inconoscibili alla mente dell'uomo" o "intenzionalmente occulte alla conoscenza altrui", così come invece accade se lo si continua ad intendere come maschera. Ė un atteggiamento, anche questo, che sottende una certa ipocrisia, quella derivata dall'incapacità spesso riscontrata in tanti spazi pub- lo che stiamo compiendo è un processo artificiale ${ }^{14}$ e di assumerne la responsabilità, con onestà intellettuale e intelligenza. Un progetto di spazio pubblico può dirsi pienamente paesaggistico anche se integralmente minerale, non è la presenza del 'verde' il discrimine quanto semmai la comprensione della natura sistemica dei luoghi, della loro storia, del Ioro metabolismo, del loro valore simbolico. Questo però rimane un concetto oscuro a molti. Ed ecco che a tentare di compensare quel recondito ma sempre presente senso di colpa nei confronti della natura, compaiono lacerti di verde, piccoli, spesso sgraziati, posticci, che a ben vedere rendono ancor più lampante la crisi della relazione essere umano - natura nei progetti e nel pensiero che li ha generati. Tali interventi, senza probabilmente avvedersene, negano infatti la stessa essenza delle piante che è quella di creare connessioni tangibili e intangibili nello spazio circostante attraverso la loro architettura e i processi che innescano sul piano biologico (Balmori, 2015; Coccia, 2016). Di essi viene esibita solo l'immagine. La relazione con la natura non può limitarsi al dato estetico-visuale, né però può essere gestita su base quantitativa-compensativa nel riguardo dei servizi ecosistemici che le piante possono offrire per il miglioramento della qualità dell'aria, la segregazione di Co2, etc. Si tratta piuttosto di operare una capriola nel nostro modo di pensare e agire dunque convintamente sulla città secondo quanto Diana Balmori ha ben sintetizzato nel suo A Landscape manifesto, soffermandosi sul ruolo cruciale dei paesaggisti (lei parla di landscape artists) i quali, afferma, "(...) need to design to allow for change while seeking a new course that enhances the coexistance of humans and the rest of nature" (punto 3) e ancora "(...) can reveal the forces of nature underlying cities, creating a new urban identity from them" (punto 14).

\section{Emergente. Una selva in movimento}

Cambiare è la cifra identitaria del mondo. Nel corso della storia l'essere umano ha modificato profon- 
damente la struttura del territorio, a varie riprese e certamente non sempre in modo dannoso (Balmori, 2010). Ciò che conferisce al cambiamento che attualmente viviamo un carattere di eccezionalità sta nel fatto che avviene nell'Antropocene, era in cui l'azione umana ha inciso talmente tanto da innescare modificazioni ecosistemiche in modo non reversibile (Crutzen, 2005) e in cui il mondo che la fisica quantistica, la teoria generale della relatività e ancor prima la termodinamica ci hanno restituito è molto più complesso di quello che credevamo (Morin, 2017). Complice la scellerata negligenza di una concezione dicotomica e riduzionista della realtà e smemorati quanto basta da non aver mantenuto nella nostra cassetta degli attrezzi il sapere sistemico del nostro passato mezzadrile, abbiamo pensato, e forse ancora pensiamo con presunzione cartesiana, che la trasformazione del mondo possa essere efficacemente operata a senso unico dall'Io allo Spazio, dalla Mente alla Natura. La realtà invece è circolare, la verità del mondo che abitiamo presuppone relazioni cicliche e complesse.

“La vera realtà (...) è mista, vaga, multidimensionale: la vera realtà è l'eco-(bio-socio-)logia complessa costituita da eco-organizzazioni biologiche e sociali nelle quali l'urbano, il rurale, il selvatico si intersecano e interagiscono con interazioni complementari, concorrenti, antagoniste e incerte" (Morin, 2008).

Dobbiamo gestire una situazione di grande delicatezza e di effettiva novità sul piano degli effetti che potremmo esperire nel breve periodo (IPCC, 2018) per cui è necessario dotarsi di strumenti e strategie che non replichino quella visione unidirezionale e lineare, ma di avere molteplici mezzi per interpretare e intervenire su processi complessi.

Secondo questa visione e già a partire dalle intuizioni della geofilosofia (Deleuze e Guattari,1994; Cacciari, 1994; Bonesio, 1997) e dalle teorie dello Spatial Turn, lo spazio ha dismesso la condizione di entità inerte per rivendicare un ruolo attivo, concrea- tivo, ci condiziona e ci plasma non meno di quanto noi facciamo con esso (Soya, 1989). Riconoscere allo spazio questo ruolo di forza motrice è un ulteriore argomento a sostegno della concezione sistemica del paesaggio. II paesaggio, tuttavia, è un particolare tipo di sistema poiché è originato da fenomeni naturali e sociali che possono essere "modellati" come sistema dall'osservatore (Von Bertalanffy, 1968), il quale dunque non è il partecipante occasionale ma contemporaneamente spettatore e attore (Turri, 2006) e il suo modellamento è quindi costitutivo dell'integrità dell'intero sistema. Maurice Merleau-Ponty in una conferenza tenuta alla Société Francais de la Philosophie di Parigi nel 1946, sosteneva che "il mondo percepito costituiva lo sfondo sempre assunto da ogni razionalità, da ogni valore ed esistenza. Un tale concetto non distrugge né la razionalità né l'assoluto. Cerca di riportarli con i piedi per terra". Questa posizione filosofica supera ogni tentativo della scienza di portarci di fronte alla dicotomia cartesiana tra Soggetto e Oggetto e restituisce una realtà relazionale come assunzione di ogni coscienza.

\begin{abstract}
"Pensare divedere ciò che è oggettivamente presente (cioè, indipendentemente dall'osservatore, non solo un rivelatore ma un creatore di realtà cognitiva) è come pensare di lavorare con un modello vuoto senza un modello cognitivo, cosa impossibile per l'attività cognitiva dei sistemi viventi e tanto più per l'uomo, il cui sistema cognitivo ha più complessità" (Minati, 2009).
\end{abstract}

Di fronte alla disgregazione del precedente ordine sensoriale la complessità induce una riforma del pensiero che porta a vedere il mondo non più scisso tra Mente e Natura, ma come "un tessuto interdipendente, interattivo e interretroattivo tra le parti e il tutto e tra il tutto e le parti" (Morin, 1999). La complessità, avverte Morin, non è però da intendersi come "parola-soluzione" quanto come "parola-problema" che ci pone di fronte a una sfida continua. 
Comprendere il paesaggio secondo il paradigma della complessità significa quindi accettare questa sfida superando il contrasto tra scienze umane e scienze naturali, che da più di un secolo si contendono il dominio del paesaggio, verso un approccio sistemico, costruttivo e, potremmo dire, cibernetico. II paesaggio è sempre concepito come il "prodotto spontaneo" di un'interazione dinamica di sistemi biologici e cognitivi. Ciò significa che il processo di organizzazione, cioè di acquisizione di nuove proprietà, di un sistema così complesso avviene in modo autonomo, inaspettato e non ripetitivo, non deducibile a stimoli esterni o alla loro attività collettiva. II modo in cui le nuove proprietà del paesaggio vengono date alle nostre percezioni 'emerge' ogni volta dall'interazione continua e dinamica dei suoi sottosistemi. Il processo per cui i sistemi complessi acquisiscono nuove proprietà prende propriamente il nome tecnico di emergenza (Minati e Pessa, 2006). II processo di emergenza può rappresentare efficacemente il modo in cui il paesaggio evolve incessantemente, è il modo in cui 'arriva a noi' e il modo in cui il nostro contributo si riflette in esso (Marinaro, 2018). Chiarire questo rapporto con l'emergenza su cui il progetto si misura costantemente ci porta ad un primo presupposto il cui scopo è fondamentale per comprendere e riconsiderare lo stesso concetto e funzionamento del processo progettuale e l'uso che ne facciamo: il progetto ha costantemente a che fare con l'incertezza. Tale incertezza non riguarda l'incertezza probabilistica degli eventi ma è 'strutturale' in quanto connessa all'atto del percepire, ovvero riguarda la gestione delle strategie mediante le quali i processi di acquisizione delle stesse proprietà emergenti vengono tradotti in modelli cognitivi da gli osservatori. Ciò significa che il paesaggio e le sue proprietà emergenti non possono essere concepiti, in maniera riduzionista, a partire solo dalla conoscenza degli elementi base del sistema per la loro composizione o regolazione, e allo stesso mo- semplice concatenazione di supposti input -output, poiché ad ogni passo del processo si ha un aumento di complessità che modifica le proprietà del sistema stesso. Poiché il paesaggio è per lo più il prodotto collettivo della percezione umana, cioè dell'interazione costruttiva tra sistemi cognitivi complessi, esso è sempre un processo evolutivo.

Questa consapevolezza, rispetto ai processi di trasformazione e innovazione dei paesaggi, non può dunque non trovare rappresentazione nel nostro agire, se di transizione ecologica vogliamo parlare.

\section{Esplicito. La città politica}

Lo spazio con cui la società attuale si confronta prevalentemente è quello della città e lo sarà sempre di più, viste le previsioni del World Urbanization Prospects 2018 delle Nazioni Unite, che attestano al 2050 una concentrazione di quasi il 70\% della popolazione mondiale in aree urbane. Confrontarsi dunque con lo spazio urbano costituisce la sfida odierna più pressante, soprattutto perché siamo chiamati a ripensarlo integralmente, ripensando la nostra relazione con esso. Nel tempo la città è sempre stata in grado di comunicare, instillare, insegnare un codice etico, di comportamento, di riflettere un ordine sociale e programmatico. Città e politica sono sempre state due facce di una stessa medaglia, basti pensare che la stessa parola, "politica" deriva da $\pi$ ó $\lambda$ s "la città stato" greca, archetipo della città moderna mediterranea che nasce dunque come forma esplicita e sentita dell'ordine sociale di una comunità, esempio perfetto di fusione tra tangibile ed intangibile. La politica è un fatto urbano, modella e al contempo emerge dalla città, attraverso di essa trasmette messaggi, impone limiti, dispiega orizzonti, schiude esperienze, educa e forma la civitas. La città ha dunque sempre trasmesso una visione, un messaggio politico. Lo si ravvisa nel castrum romano, nella generosità degli sporti di gronda medicei in alcune vie del centro di Firenze e dei portici del centro di Bologna, nella specializzazione mercantile della rete di canali di Am- 
sterdam, nell'impeto sansimonista dei grand boulevards Hausmanniani, ecc. La città fino all'età moderna trovava la sua compiutezza semantica e formale attraverso il concetto di limes, nel confine tra un dentro e un fuori, tra un centro e una periferia, tra città e territorio, limiti coerentemente riflessi dalla suddivisione legale, politica del mondo. Nell'era capitalistica, l'egemonia progressiva del mercato nell'organizzazione del lavoro, della produzione, degli scambi commerciali, della mobilità, nel giro degli ultimi 70 anni ha modificato radicalmente la nostra società e la nostra cultura inducendo un cambiamento anche nel codice genetico della città. La connettività è divenuto il valore preponderante traghettandoci da un mondo in cui la "geografia era destino" ad uno dominato e riscritto interamente dalle supply-chains ${ }^{15}$. L'età dell'organizzazione del mondo secondo lo spazio politico ha ceduto il passo a quella secondo lo spazio funzionale in cui non solo le linee che ci connettono sono molte di più di quelle che ci dividono ma soprattutto, nella loro fitta interrelazione, sono molto più importanti dei singoli punti che uniscono. II cambiamento radicale che la connettività ha innescato, e che ha travolto il territorio, sta proprio in questo spostamento di accento dai poli alle infrastrutture (siano esse tangibili o intangibili) e nella gerarchia di valore che quest'ultime hanno assunto nell'economia mondiale. Emblematico è l'esempio del ponte di Øresund:

"Legate dal doppio percorso autostradale e ferroviario del ponte di Øresund, le economie di Copenaghen e Malmö sono diventate cosi connesse che molti parlano oggi di un'unica 'KoMa'. L'aeroporto di Copenaghen ora è più vicino per gli abitanti di Malmö che non quello della loro stessa città, e i tassisti svedesi vi hanno una propria area riservata. I paesi baltici tentarono di formare un'intesa poco dopo la prima guerra mondiale ma furono divisi dall'espansionismo sovietico. Ora, un secolo dopo, una molto più larga Unione baltica ha preso forma, dalla Norvegia alla Lituania, ed è direttamente connessa all'Europa occidentale proprio tramite il ponte di Øresund" (Khanna, 2016, p.50).
II portato di trasformazioni come queste, della quali abbiamo innumerevoli esempi in tutto il globo, riverbera sonoramente sul piano intangibile, poiché induce una mutazione della percezione della popolazione, riscrive codici di identità e appartenenza ai luoghi e rivoluziona la relazione con la geografia. “(...) La connettività non ha a che fare con una qualche specie di dismissione del sapere geografico, ma con un suo uso migliore. Essa muta la nostra percezione di cosa costituisca una regione naturale" (Khanna, 2016). Ecco che anche il ragionamento sulla connettività porta nuovi argomenti a favore di una riformulazione del discorso su cosa sia veramente 'naturale' e cosa sia 'artificiale' rivelando la necessità di adottare un approccio sistemico e complesso piuttosto che oppositivo e riduzionista. $E$ in questo contesto comprendiamo anche come la dialettica tra tangibile e intangibile sia pressoché incessante, generativa della nostra realtà.

Le supply-chains, entità perlopiù intangibili, sono oggi il principale attore sulla scena mondiale, responsabili della sempre maggiore complessità e polimorfia della selva e della progressiva sostituzione del genius loci della città con il genius loci dell'economia globalizzata. Ciò ha avuto ovvie e incessanti ripercussioni sul territorio che abitiamo, adoperando ovvero una trasformazione capillare che, attraverso meccanismi diffusi e spontanei, si è data per erosione, intrusione, suddivisione, frammentazione, riduzione, eliminazione, concentrazione, interruzione dei processi ecologici, destrutturazione, deconnotazione $^{16}$ dell'ordine precedente, senza ovvero nella maggior parte dei casi esser guidata da un disegno complessivo, da una visione strategica integrata, ovvero senza un progetto.

L'interpretazione di questo processo ha via via riempito le nostre biblioteche di visioni e letture critiche dei fenomeni in atto nei paesaggi che conoscevamo, i cui contorni si sono liquefatti, la cui divaricazione ha prodotto scarti, paesaggi terzi, junkspaces (Koolhas, 2006), dross-scapes, vuoti ed eterotopie, etc 
... La dicotomia Castelliana tra lo "spazio dei luoghi" e lo "spazio dei flussi" e la gerarchia funzionale e di valore che il mercato vi ha impresso, ha mutato in modo decisivo la percezione cognitiva delle popolazioni incidendo in modo pesante nella relazione tra le comunità e il proprio territorio e andando a scapito dell'integrità di moltissimi paesaggi, che sono stati quasi svuotati da dentro (Bonesio, 1997).

Abbiamo erroneamente pensato che il Mercato, con le sue leggi e le sue dinamiche, potesse farsi garante dell'ordine della Informational City, sostituendosi alla politica. Questa credenza si è rivelata fallace non solo sul piano intangibile dei valori e dei diritti, ma ha intaccato fortemente la struttura territoriale, ha prodotto condizioni e spazi che hanno acuito quando non hanno generato nuove pesanti diseguaglianze. Pensiamo ad esempio al fenomeno del digital divide, o a quello che Mimi Sheller definisce delle "mobilità ineguali"177 che oggi sono cruciali per comprendere i limiti della transizione alla sostenibilità (Sheller, 2018).

"When a young woman, a black teenager, lesbian, gay, bisexual, transgender, queer (LGBTO) couple are unable to walk down the street or board public transportation without being harassed, there is an injustice of social exclusion which occurs through a restriction on their mobility. When a child, or college student, or underpaid bicycle-delivery person cannot ride safely in the streets without the likelihood of being hit bye careless car driver, there is an injustice of social protection that distributes the harms of automobility upon the most vulnerable. (...) When a city and its surroundings are built in a way that makes most people living in them automobile dependent, there is an injustice for those without automobile and for those affected by the air pollution, traffic and potential crashes created by excessive automobile dependency. When a urban redevelopment project put a multi-line through a working-class neighbourhood dividing it in a half and obliterating homes and businesses or when a new light-rail line bypasses poorer area and brings the service only to the better off, there is an injustice enacted in spatial organization. (...) Mobility justice is not only personal or infrastructural, but also linked to national scale issues of migrant justice and transnational scale issues of climate justice" (Sheller, 2020).
Esperiamo sempre più spesso gli effetti degenerativi che la connettività ha impresso nella forma della città ed è una condizione che non solo dobbiamo ma possiamo efficacemente ribaltare in quanto la connettività è invece portatrice potenziale di innumerevoli valori positivi ad esempio nella sua accezione di solidarietà.

Dunque progettare la città della transizione ecologica oltre a presupporre un cambio di paradigma nel modo di vedere il mondo, nel concepire la relazione tra essere umano e natura, ha anche a che fare con la necessità di sovvertire questa preminenza del mercato sulla politica, per restituire agli interventi nella città un carattere di lungimiranza e validità generale, emancipandoli dalla moda e conferendo loro capacità di rappresentazione e di indirizzo dei valori e dei bisogni del contemporaneo, per come lo intendeva Agamben (2008). II Mercato va regolato, ed ecco che "Progettare la transizione ecologica"18 assume il carattere di un progetto politico che è insieme culturale ed educativo.

Tornando ad esempio sulla spinosa questione della transizione energetica -tutela del paesaggio di cui si è fatto cenno prima: lasciare di fatto allo Stato un mero ruolo di controllo (si veda la recente creazione della 'Super-soprintendenza' da parte del MiBACT) e delegare perlopiù alla sola iniziativa privata il compito di proporsi sul territorio per la realizzazione di impianti FER, è assurdo. Testimonia una rinuncia al progetto e una totale assenza di visione. Oggi vi è una necessità estrema di tornare a pianificare, non è pensabile affrontare la sfida della transizione energetica delegando ancora una volta al mercato il compito di guidare la trasformazione. Serve un disegno, servono Piani e Progetti ed è necessario che abbiano al loro centro il paesaggio della CEP.

Appresa la lezione ecologica, l'elemento innovativo sta nel comprendere che questa rivoluzione non è detto che debba essere compiuta solo attraverso norme e leggi, ma può efficacemente essere innescata e condotta attraverso un lavoro sullo spazio, 
su quella originaria "selva", facendo del paesaggio il mezzo, il depositario e al contempo l'attore capace di coadiuvare, rendere esperibile il cambiamento per poi spazializzarlo appieno.

Detto ciò, non si può pensare di delegare il compito di guidare il processo di transizione ecologica alla sola classe politica per come sino ad oggi è composta e intesa. La sfida globale richiede sforzi congiunti e nuove competenze capaci di dialogare a partire dal lavoro che ognuna di esse svolge attivamente nel suo campo. È dunque il lavoro, l'esperienza diretta sul campo a farsi base per il dialogo, a costruire nuovi orizzonti di senso, nuova evidenza. Augurarsi infatti che siano i segnali del cambiamento climatico da soli a dare la misura della gravità della condizione in cui ci troviamo è una negligenza che non possiamo permetterci. II fenomeno del negazionismo come strategia estrema di difesa di assetti e poteri consolidati ha già dato ampia prova della sua pervicace pervasività, soprattutto oggi che la crisi culturale fornisce un humus perfetto.

Forte della lezione di Tocqueville: "Un'idea semplice, ma falsa, avrà sempre più peso nel mondo di un'idea vera, ma complessa", il negazionismo climatico promosso dalle lobby del fossile (Levantesi, 2021), ha nel tempo affinato la sua capacità comunicativa conducendo a situazioni paradossali di enorme gravità sul piano politico internazionale come l'uscita dell'America dall'Accordo di Parigi voluta da Trump nel 2017. Non ultimo con la pandemia abbiamo assistito a nuove forme di negazionismo miste a teorie complottiste serpeggianti. Ciò per dire che il tema dell'evidenza è tutt'altro che scontato e rivela la sua centralità per affrontare il processo di transizione ecologica. C'è bisogno di un'evidenza che possa essere esperita collettivamente. In tal senso dunque assume ancora maggior rilievo quel ruolo di mediazione che il progetto di paesaggio può e deve svolgere tra il piano dell'intangibile e quello del tangibile e viceversa. In quanto esso può aiutare la popolazione a rinsaldare il legame con il territorio, farsi promotore del re-incontro con i luoghi (Nogué, 2016) per costruire nuovi orizzonti di senso e comunità fondate su nuovi valori condivisi. Si torna dunque a ribadire l'importanza di concepire il progetto di paesaggio come dispositivo rivelatore, capace di rendere esplicito ciò che emerge dalla rinnovata relazione tra esseri umani e natura per creare nuova evidenza. Agire sulla dimensione tangibile perché essa riverberi ed informi quella intangibile, perché inneschi quel processo di realizzazione, di progressivo avvicinamento dello scenario catartico, assume eguale importanza dell'azione legislativa tout court. Sgombrato il campo dalla 'cromoterapia', il progetto di paesaggio, acquisisce lo status di atto politico fortemente caratterizzato e caratterizzante, rivelando enormi potenzialità nel farsi promotore della transizione ecologica oltre che per un fatto costitutivo (il concetto di paesaggio implica la transizione ecologica) anche sul piano pratico operativo, poiché essendo un progetto di relazioni, è capace a gestire e mai banalizzare la complessità.

Abbiamo bisogno di un nuovo paradigma di città. Una città selvatica, non più antropocentrica. Scevro dallo spettro del dominio cartesiano del solo essere umano, lo spazio urbano diverrebbe uno spazio liberato, capace di aprire gli orizzonti del possibile, accogliere l'imprevisto, dialogare e farsi scenario dell'emergente ispirato da una tolleranza creativa. È il caso di progetti come il Jardins du Tiers-Paysage di Gille Clément e Coloco (2011), pensato e realizzato per il tetto della vecchia base Sottomarina di Saint Nazaire, che Manuel de Solà Morales chiamava "il mostro" oppure del progetto di rinaturalizzazione del Fiume Aire, progettata e diretta da George Descombes e ancora in corso. La 'Città Selvatica' è la selva vissuta, domesticata, riscritta.

"una chimera, che sfida le leggi dell'ordine costituito della separazione; un mostro, talvolta maligna talvolta benefico, che mescola e confonde elementi che non potrebbero essere legati fra loro; [...] La città selvatica non si nutre di alcuna pretesa prometeica, cosi 
come, viceversa, non richiede alcuna rinuncia al progetto. Al contrario, lo auspica e lo attende" (Metta, Olivetti, 2019).

Serve una città produttiva. Al pari della politica energetica è oggi necessaria una politica industriale, la sfida è che siano entrambe paesaggistiche. Immagino una città che non espella i centri di produzione ma che agisca convintamente per incorporare la produzione di energia e l'industria lavorando sul concetto stesso di luogo di produzione, eliminando cioè quei difetti e quegli impatti connaturati al suo passato di soggetto inquinante retaggio dell'economia fossile, e conferendo attraverso il progetto una nuova estetica e pari dignità ai luoghi della produzione. Guardiamo ad esempio al progetto del Parc del Garraf dello studio Batlle i Roig, dove la discarica di una metropoli è diventata un parco e produce energia rinnovabile!

Una città creativa e solidale. Lo spazio pubblico della città del XXI secolo deve poter essere accogliente, duttile e sicuro ma soprattutto deve essere vario e stimolante per dare spazio alle molteplici attività che le persone possono svolgere o ancor meglio istigarle. Flessibile non significa però asettico. Abbiamo bisogno di uno spazio pubblico non banale, che stimoli l'inventiva, la creatività e che si faccia possibilmente esempio per innescare comportamenti etici, educare alla tolleranza, alla pacifica convivenza e al dialogo.
Progetti come Superkilen dello studio Topotek a Nørrebro Copenhagen (2012) hanno lavorato sul tema dell'inclusione e del sincretismo creando una nuova simbologia, democratica e non discriminatoria dello spazio pubblico. È un progetto di paesaggio pur avendo piantato pochissimi alberi! Uno spazio pubblico che stimoli l'adozione di buone abitudini, la lettura, la sosta, il gioco, penso alle sedie verdi dei parchi parigini, ai giochi fissi da strada, alla soglia di contemplazione recuperata ad Auckland con la North Warf Promenade e il Silo Park dello studio TCL. Una città che più che 'mettere la scuola al centro' diventi come una 'grande scuola a cielo aperto' (soprattutto oggi in un Paese come il nostro che ha progressivamente mortificato la scuola pubblica).

"La scuola nonè un edificio. Deve funzionare come sistema integrato alla città in un nuovo quadro di economia sociale e solidale. è questa la visione che manca ... oltre a tutto quello che manca. (...) Nel Pnrr, ridisegnare il rapporto tra scuola e territorio non è prioritario, ma neppure incluso tra le necessità della scuola contemporanea. Ed è questa una lacuna gravissima che si riflette a scala urbana, anche perché la scuola è il soggetto istituzionale pubblico più diffuso nel territorio. Questa mancanza non è, semplicemente, da attribuire a un'incapacità politica di lettura e interpretazione del macro-tema scuola/città. Ha radici molto più profonde che derivano dall'assenza di un'economia sociale e solidale, un modello di sviluppo orientato ai bisogni degli individui più che al profitto" (Femia, 2021). 
Molti ancora possono essere gli attributi della città del XXI secolo, mutevoli ed evolutive dovranno essere le sue caratteristiche all'insegna di una maggiore democrazia, solidarietà e creatività. Le riflessioni espresse in questo saggio aprono la strada al dibattito proponendosi di mettere a sistema le questioni attuali in tema di governo del territorio, tutela e valorizzazione del paesaggio e transizione ecologica, sottolineando la loro logica e necessaria interconnessione e chiamandoci ad una maggiore responsabilità etica professionale e soprattutto politica. Tanti possono essere i punti di partenza, molte le occasioni in cui riconferire dignità al progetto, sia che si parta dal piano tangibile dello spazio sia che si inizia da quello intangibile delle norme, dal momento che la realtà emerge dalla relazione continua tra i due piani. Ripensare gli stardard urbanistici (definiti nel 1968) adeguandoli alle sfide attuali della transizione ecologica oltre ad essere un imperativo urgente offrirebbe ad esempio l'occasione di un rinnovamento radicale dei principi che informano gli strumenti per la pianificazione e che ispirano i progetti. Nei nuovi standards vicino ai metri quadrati e al tempo per la residenza, perché non potrebbero comparire quote di energia da FER, metri cubi d'acqua potabile e di ossigeno, km di trasporto pubblico, metri quadrati di spiaggia libera e di mare limpido, GB di connessione internet, ore di silenzio, ...?

\section{Note}

${ }^{1}$ Canto I. la Divina Commedia, tra le più sublimi e rappresentative narrazioni di processi di transizione che la nostra letteratura ci offre, si apre con un paesaggio, quello appunto della "selva, selvaggia, aspra e forte". Un micro-cosmo linguistico potentissimo che con sole quattro parole riesce a descrivere e comunicare la multi-sensorialità dell'esperienza del paesaggio e la sua profondità semantica attraverso un gioco di assonanze, allitterazioni, asindeto, sinestesia e allegoria. La selva oscura dantesca è l'allegoria del sonno della ragione, dello smarrimento, del peccato che conduce alla perdizione (non poi così distante dallo scenario dei $+2^{\circ} \mathrm{C}$ ).

${ }^{2}$ Accordo di Parigi. Convenzione quadro delle Nazioni Unite sui cambiamenti climatici.

${ }^{3}$ Presentato nel 2019 || Green Deal Europeo è il programma che i 27 Stati membri dell'UE hanno redatto per trasformare l'UE nel primo continente a impatto climatico zero entro il 2050, parte integrante della strategia della Commissione per attuare l'Agenda 2030 e gli obiettivi di sviluppo sostenibile delle Nazioni Unite. Parte integrante della strategia e tappa fondamentale per la sua attuazione è il Patto Europeo per il Clima siglato nel giugno 2021. Detto anche FitFor55, con le sue 12 proposte legislative è di fatto la prima legge quadro europea per contrastare i cambiamenti climatici e raggiungere l'obiettivo di una riduzione delle emissioni di gas a effetto serra per il 2030 di almeno il 50-55 \% rispetto ai livelli del 1990. 12. Nella roadmap del Green European Deal vi sono anche una nuova strategia industriale, forestale, per l'economia circolare (Farm to Fork' strategy), per la mobilità sostenibile, per la biodiversità, per la finanza sostenibile e per un ambiente privo di sostanze tossiche. Concorrono alla sua realizzazione un terzo degli 1,8 trilioni di euro del NextGenerationEU Recovery Plan e il budget settennale dell'UE.

${ }^{4}$ Appellativo che deriva direttamente dalla teoria della "decrescita serena" dell'economista e filosofo francese Serge Latouche. 
${ }^{5}$ Parola greca antica che indica la forza della natura e il principio ordinatore del cosmo che poi ha assunto varie sfaccettature di significato in filosofia e nella scienza.

${ }^{6}$ La bruttezza, l'odiosità della selva nella divina commedia si spiegano come esito di un processo di progressiva alienazione di questi spazi naturali dal circuito di vita delle persone che ha inizio con la dissoluzione progressiva dell'impero romano e si rafforza in epoca medievale. In questi spazi, in cui la natura ha ripreso il sopravvento, domina il pericolo e sono contrapposti all'ordine urbano. La selva, secondo la visione Dantesca, è allegoria del peccato di una condizione di perdizione e di smarrimento, questa visione si fissa nell'immaginario collettivo (ne era in qualche modo già essa stessa espressione) condizionando pesantemente l'approccio e la gestione della parte tangibile di questi beni. Si vedano a tal proposito La città nella storia d'Europa (Benevolo) e Storia della Bruttezza (Eco).

' $\mathrm{E}$ più in generale diremmo 'il paesaggio' in toto per come viene definito dalla CEP.

${ }^{8}$ Riconoscendo che il paesaggio è in ogni luogo un elemento importante della qualità della vita delle popolazioni nelle aree urbane e nelle campagne, nei territori degradati, come un quelli di grande qualità, nelle zone considerate eccezionali, come in quelle della vita quotidiana". Preambolo. Convenzione Europea del Paesaggio, Firenze 2000.

${ }^{9}$ L'identificazione con la sola componente tangibile ha dei risvolti molto rilevanti e sostanzia quasi un paradosso. Inteso come luogo materiale, concreto, perfetto che occupa materialmente uno spazio e che per esistere deve manifestarsi ai sensi quel paesaggio della transizione ecologica diventa 'esclusivo' perché si definisce a partire dalla differenza che si riscontra rispetto a ciò che esiste, se non si vede...non c'è (!), e dunque 'si allontana', viene proiettato in avanti in un tempo e un luogo che dovranno venire, che appartengono alla sfera del desidero. Solo Tangibile il paesaggio diventa Utopico.
${ }^{10}$ Domandarsi il perché ciò infondo accada è importante. Domandarsi perché vi sia questa implicita percezione comune. Da cosa scaturisce se non forse dal rendersi conto di una mancanza di messaggio, giustificazioni, valori, ragioni che esulino da quelle dell'interesse e del mercato e che siano effettiva espressione dei desiderata, delle necessità, dell'identità della comunità? Ė come se si fosse diffusa una certa sfiducia nel piano e nei progetti come strumenti efficaci per condurre trasformazioni congrue e rappresentative, ed è una dinamica della quale come progettisti bisogna prendere atto per riformulare risposte convincenti.

${ }^{11}$ Ad oggi i piani paesaggistici adeguati alle prescrizioni del Dlgs 42/2004 il Codice dei beni culturali e del paesaggio (artt. 135 e ss. del decreto legislativo n. 42/2004 e s.m.i.) sono quelli della Sardegna (2006), della Puglia (2015), della Toscana (2015), del Piemonte (2017) e del Friuli - Venezia Giulia (2018).

12 II Piano Nazionale integrato Energia e Clima redatto dal Ministero dello Sviluppo Economico con il Ministero dell'Ambiente e della tutela del territorio e del mare e il Ministero delle Infrastrutture e dei Trasporti, redatto in attuazione del Regolamento (UE) 2018/1999 recepisce le novità contenute nel Decreto Legge sul Clima nonché quelle sugli investimenti per il Green New Deal previste nella Legge di Bilancio 2020. Nelle 294 pagine del piano la parola "paesaggio" compare quattro volte sempre con una pesante accezione statico conservatrice e mai in ottica processuale e progettuale. II paesaggio nello PNIEC mantiene l'accezione oggettuale inerte di "sfondo" delle trasformazioni che devono pertanto dotarsi dell'adeguata cosmesi. È significativo che alla sua redazione non abbia partecipato il MiBACT, permane almeno il dubbio che qualcosa di più sostanzioso in tal senso potesse essere fatto. Come paesaggisti, promotori della CEP, possiamo però oggi accontentarci di questo? 
${ }^{13} \mathrm{Si}$ ha dunque l'esigenza concreta di fare chiarezza su cosa siano il paesaggio e il progetto di paesaggio, con un messaggio che raggiunga un pubblico il più vasto possibile, i cittadini ma soprattutto le amministrazioni, gli enti, ecc. per procedere ad una effettiva attuazione, prima ancora che ad una auspicabile attualizzazione, della CEP. In risposta a tale bisogno si pone la recente operazione della Società Scientifica Italiana di Architettura del paesaggio, IASLA, con la traduzione in lingua italiana delle Linee guida per l'attuazione della Convenzione Europea del Paesaggio del 2008 (Recommendation CM/Rec(2008)3 of the Committee of Ministers to member states on the guidelines for the implementation of the European Landscape Convention) che, fino ad oggi non avevano mai trovato una traduzione ufficiale nel nostro Paese. Si tratta di un primo importante passo verso la condivisione di un linguaggio comune e ovviamente verso la transizione. Attualmente in corso di pubblicazione, il libro affianca al testo tradotto delle linee guida una serie di note, riflessioni critiche che accompagnano la traduzione per renderla ancor più efficace ed esplicita nell'evidenziare e dare risposta a dubbi o cortocircuiti rispetto ad alcune questioni che riguardano il paesaggio oltre che per dare indirizzi nell'attuazione della CEP. Concepito come preludio ad un lavoro critico ancora più cospicuo, il lavoro è stato curato da Daniela Colafranceschi, Gabriele Paolinelli, Fabio di Carlo, Ludovica Marinaro, Emma Salizzoni, Maria Livia Olivetti e Matteo Aimini.

${ }^{14}$ Se assumiamo la definizione di paesaggio introdotta dalla CEP (2000), esso si può a ragione intendere come la risultante di un processo artificiale, cioè ottenuto ad arte e per opera dell'essere umano. Vi sono poi altre brillanti visioni che sostengono questa lettura, come quella di Alain Roger (1997) che indica l'origine culturale del paesaggio come doppio processo di 'artializzazione' in situ e in visu ( $\mathrm{Pa}-$ olinelli, 2009). L'arte si libera dal giogo di essere imitazione della natura e rivendica su essa un ruolo creativo, generatore. La Natura che conosciamo può dunque prescindere ontologicamente dall'azione antropica? L'affermazione di Roger in questo è tranchant, "la natura è nata dalla nostra mente". Esistono dunque paesaggi non artificiali?

${ }^{15}$ Parag Khanna, stratega geopolitico indiano, definisce le supply chains come "l'ecosistema completo di produttori, distributori e venditori che trasformano materiale grezzo (dalle risorse naturali alle idee) in beni e servizi erogati alla gente in qualsiasi parte del mondo" (2016).

${ }^{16} \mathrm{Si}$ tratta di tutte le dinamiche di trasformazione delle quali si deve tenere conto e descrivere nel redigere la Relazione Paesaggistica, quell'elaborato preposto a verificare e coadiuvare l'integrazione paesaggistica di opere di trasformazione del territorio. Tali dinamiche sono state così definite e compaiono tra i criteri guida per lo svolgimento delle analisi dei contesti ante e post trasformazione nel Decreto del presidente del Consiglio dei ministri 12 dicembre 2005. Individuazione della documentazione necessaria alla verifica della compatibilità paesaggistica degli interventi proposti, ai sensi dell'articolo 146, comma 3, del Codice dei beni culturali e del paesaggio di cui al decreto legislativo 22 gennaio 2004, n. 42. Nota 8 all'articolo 3.2 comma 3.

${ }^{17}$ La ricerca della sociologa statunitense Mimi Sheller approfondisce proprio i problemi della transizione ecologica in relazione alla 'automobilità' e alle infrastrutture di trasporto e urbane, lega insieme i problemi di giustizia sociale, di ingiustizie globali, le teorie postcoloniali e decoloniali, con le questioni del potere della politica e della mobilità. ${ }^{18}$ Questo il titolo di un nuovo progetto corale itinerante che ho il piacere di curare per il Think Tank TES - Transizione Ecologica Solidale e che mette al centro della sua riflessione in vari contesti territoriali italiani il tema del progetto della transizione ecologica secondo un approccio sistemico, transisciplinare e creative commons. Recensioni dei primi due eventi costruiti a Livorno e a Torino si possono trovare sul sito web dell'associazione TES. 


\section{Bibliografia}

AA.VV. 2009. The Spatial Turn: Interdisciplinary Perspectives, ed. B.Warf and S.Arias, Routledge, London-New York.

Agamben G. 2008. Che cos'è il contemporaneo? I sassi, Nottetempo, Milano.

Balmori D. 2010. A landscape manifesto. Yale University Press, New Haven, Connecticut.

Benevolo L. 2004. La città nella storia d'Europa. Editori Laterza, Roma Bari.

Bonesio L. 1997 Geofilosofia del paesaggio. Mimesis, Milano.

Cacciari M. 1994. Geofilosofia dell'Europa. Adelphy Edizioni, Milano.

Coccia E. 2016. La vie des plantes. Une métaphysique du mélange. Bibliothèque Rivages, Paris.

Crutzen P. 2005. Benvenuti nell'Antropocene. L'uomo ha cambiato il clima, la Terra entra in una nuova era. Mondadori, Milano.

Decreto del presidente del Consiglio dei ministri 12 dicembre 2005. Individuazione della documentazione necessaria alla verifica della compatibilità paesaggistica degli interventi proposti, ai sensi dell'articolo 146, comma 3, del Codice dei beni culturali e del paesaggio di cui al decreto legislativo 22 gennaio 2004, n. 42. (G.U. n. 25 del 31 gennaio 2006).

Deleuze G., Guattari F. 1994. Geofilosofia. II progetto nomade e la geografia dei poteri. Mimesis, collana millepiani, Milano.

European Commission, 2016. Accordo di Parigi. GU L 282 del 19.10.2016, pagg. 4-18. < https://eur-lex.europa.eu/legal-content/IT/TXT/?uri=CELEX:22016A1019(01)>.
European Commission, 2019. Comunicazione Della Commissione Al Parlamento Europeo, Al Consiglio, Al Comitato Economico E Sociale Europeo E Al Comitato Delle Regioni. II Green Deal europeo. COM/2019/640 final. <https://eurlex.europa.eu/legal-content/IT/TXT/?qid=1576150542719 Euri=COM\%3A2019\%3A640\%3AFIN>.

Femia A. 2021. SCUOLA SOCIAL IMPACT: Far ripartire il Paese dalla scuola. 500x100 Publisher.

IPCC, 2018: Clobal Warming of $1.5^{\circ}$ C. An IPCC Special Report on the impacts of global warming of $1.5^{\circ} \mathrm{C}$ above pre-industrial levels and related global greenhouse gas emission pathways, in the context of strengthening the global response to the threat of climate change, sustainable development, and efforts to eradicate poverty. Masson-Delmotte, V., P. Zhai, H.-O. Pörtner, D. Roberts, J. Skea, P.R. Shukla, A. Pirani, W. Moufouma-Okia, C. Péan, R. Pidcock, S. Connors, J.B.R. Matthews, Y. Chen, X. Zhou, M.I. Gomis, E. Lonnoy, T. Maycock, M. Tignor, and T. Waterfield (eds.). In Press. < https:// www.ipcc.ch/site/assets/uploads/sites/2/2019/06/SR15 Full_Report_Low_Res.pdf > 16.12.2021.

Khanna P. 2016. Connectography. Le mappe del futuro ordine mondiale. Fazi Editore, Roma.

Koolhas R. Junkspaces. Quodlibet, Roma.

La Pietra U. 2015. II verde risolve. Dal giardino delle delizie al nostro verde quotidiano 1980-2014. Corraini Edizioni, Mantova.

Levantesi S. 2021. I Bugiardi del Clima. Potere, politica, filosofia di chi nega la crisi del secolo. Editori Laterza. Roma, Bari.

Marinaro L., 2018, Design in Emerging Landscapes. Acting on cultural metabolism of Mediterranean port cities. Tesi di dottorato in Architettura. Università degli studi di Firenze <https://flore.unifi.it/handle/2158/1131371?mode $=$ full.1522 $>$. 
Marinaro L., 2018, Paesaggi emergenti sui bordi del Mediterraneo. L'immagine dei grandi porti commerciali nella città dell'era globale in Spazi Liquidi, Paesaggi Ibridi. Una strategia progettuale paesaggistica per la rigenerazione urbana del distretto industriale di Sant'Agostino E. Falqui Ed. DIDAPRESS, Firenze. (pp. 89-100).

Merleau-Ponty M., 1964, L'oeil et l'esprit. Folio Essais. Editions Gallimard, Paris.

Metta A., Olivetti M. 2019. La città selvatica. paesaggi urbani contemporanei. Libria, Melfi.

Minati G., Pessa E. 2006. Collective beings. Springer, New York.

Ministero dello Sviluppo Economico, Ministero dell'Ambiente e della Tutela del Territorio e del Mare, Ministero delle Infrastrutture e dei Trasporti. 2019. Piano nazionale integrato perl'energia e il clima. (Dicembre 2019) < https:// www.mise.gov.it/images/stories/documenti/PNIEC_finale_17012020.pdf> 16.12.2021.

Morin E., 1999, La testa ben fatta. Riforma dell'insegnamento e riforma del pensiero, Editore Cortina Raffaello, Milano.

Morin E. 2008. II metodo. La natura della natura (Vol. 1). Cortina Raffaello Editore.

Morin E. 2017. La sfida della complessità. Editore Le Lettere, collana Gaiamente, Firenze.

Nogué J. 2016. El reencuentro con el lugar: nuevas ruralidades, nuevos paisajes y cambio de paradigma. Documents d'Anàlisi Geogràfica 2016, vol. 62/3. pp.489-502.

Pallasmaa J. 2005. The Eyes of the Skin: Architecture and the Senses. John Wiley \& Sons Ltd.

Paolinelli G. 2018. Progettare trasformazioni dei paesaggi nel mondo che cambia. Didapress, Firenze.
Sheller M. 2018. Mobility Justice: The Politics of Movement in an Age of Extremes. Verso, London.

Sheller M. 2020. Mobility Justice in Handbook of Research Methods and Applications for Mobilities. Büscher M., Freudendal-Pedersen M., Kesselring S., Grauslund Kristensen N. Eds. Handbooks of Research Methods and Applications series. Edgaronline. <https://www.elgaronline.com/ view/edcoll/9781788115452/9781788115452.00007.xml> 15.12.2021

Sieni V. 2021. Preistorico < http://www.virgiliosieni.it/ preistorico-virgilio-sieni/> 10.12.2021.

Soja E.W. 1989. Postmodern Geographies, Verso, London-New York.

Venturi Ferriolo M. 2009. Percepire paesaggi. La potenza dello sguardo. Bollati Boringhieri.

Von Bertalanffy L., 2004. General System Theory: Foundations, Development, Applications. (edizione originale 1968) New York, George Braziller, prefazione di Minati G. alla ripubblicazione della versione italiana, Oscar Saggi Mondadori, Milano.

Zagari F, Di Carlo F. 2016. II Paesaggio come sfida. II progetto. Librìa, Melfi.

Zanchini E. 2021. La Transizione ecologica passa dal Ministero della Cultura. Quotidiano Domani. 02.03.2021. 